\title{
Characteristics of the viromes in the pelagic zone of Lake Baikal
}

\section{Potapov S.A. ${ }^{1 *}$, Tikhonova I.V. ${ }^{1}$, Krasnopeev A.Yu. ${ }^{1}$, Kabilov M.R. ${ }^{2}$, Tupikin A.E. ${ }^{2}$, Chebunina N.S. ${ }^{1}$, Zhuchenko N.A. ${ }^{1}$, Belykh O.I. ${ }^{1}$}

\author{
${ }^{1}$ Limnological Institute Siberian Branch of the Russian Academy of Sciences, Ulan-Batorskaya Str., 3, Irkutsk, 664033, Russia \\ ${ }^{2}$ Chemical Biology and Fundamental Medicine Siberian Branch of the Russian Academy of Sciences, Lavrentiev Avenue 8, Novosibirsk \\ 630090, Russia
}

\begin{abstract}
For the first time, we characterized viromes from the epipelagic zone of three Lake Baikal basins. We determined alpha and beta diversity of viral communities, carried out functional annotation of reads, built whole genome sequences of viruses, detected auxiliary metabolic genes, and evaluated environmental factors affecting the quantitative and qualitative characteristic of viral communities.
\end{abstract}

Keywords: Viromes, Lake Baikal, High Throughput Sequencing, Biodiversity

\section{Introduction}

Viruses are obligate intracellular parasites consisting of one or two RNA or DNA molecules that are enclosed in a protein shell (capsid). Some viruses have an additional membrane lipid shell. In the aquatic environment, viruses are present both in the form of free particles and inside the host cell. Viruses of eukaryotes, archaea and bacteria represent the viral communities of aquatic ecosystems. Viruses of the latter two domains are usually called phages. Viruses play a key role in the regulation of the size and structure of microbial communities, causing the death of archaea, bacteria and algae (Weitz and Wilhelm, 2012).

The absence of a universal marker for the search and identification of viruses extremely complicates the study of the genetic diversity of viral communities. In recent years, methods of high throughput sequencing of metagenomics communities have allowed avoiding the use of specific markers and have been successfully applied for the study of biodiversity, structure and functional potential of viral communities in both sea and fresh waters (Watkins et al., 2015; Skvortsov et al., 2016; Gong et al., 2018). This study presents for the first time the results of genetic analysis of DNAcontaining viruses in the plankton from three basins of Lake Baikal.

\section{Material and methods}

Water samples with a volume of $\sim 25 \mathrm{~L}$ were collected in 2018 on 22 March, $7 \mathrm{~km}$ from the Listvyanka settlement (BVP1); 8 June, $3 \mathrm{~km}$ from Listvyanka
(BVP2); 31 May, $3 \mathrm{~km}$ from the Turka settlement (BVP3); 4 June, $3 \mathrm{~km}$ from Elokhin Cape (BVP4); and 27 September at the central station of the Listvyanka settlement - Tankhoy settlement section (BVP6). From each layer $(0,5,10,15,20,25$, and $50 \mathrm{~m}), 3.5 \mathrm{~L}$ were sampled and then mixed to obtain an integrated sample of the 0-50-m layer. Further processing of viromes, chemical analysis and determination of the amount of viral particles and bacteria were carried out as previously described (Potapov et al., 2019).

\section{Results}

In total, the samples from Lake Baikal revealed 21 families of viruses that infect a wide range of hosts: bacteria, algae, birds, fish, insects, humans, etc. The families Myoviridae, Siphoviridae, Podoviridae, Phycodnaviridae, and Poxviridae comprised $97 \%$ of all identified families. The bulk of the viral sequences belonged to tailed bacteriophages of the order Caudovirales. Among them, phage of the family Myoviridae prevailed. The phages of the family Siphoviridae were subdominants. Podoviridae were minor. In virome sampled $3 \mathrm{~km}$ from Elokhin Cape, there were many unclassified sequences determined up to domain and fewer number of sequences of the family Podoviridae compared to other viromes. Viral sequences in viromes, despite their considerable diversity, were similar in taxonomic composition. In all viromes, bacteriophages of the order Caudovirales dominated.

In the investigated samples, among all hits belonging to species, the majority was classified as cyanophages: BVP1 - 38.7\%, BVP2 - 53.4\%, BVP3 
- 29.6\%, BVP4 - 33.2\%, and BVP6 - 31.6\%. They represented phages that infect members of the genera Synechococcus, Prochlorococcus and Phormidium as well as species Microcystis aeruginosa.

Values of the Shannon index varied from 3.737 to 4.393, and Simpson's diversity index - from 0.916 to 0.976 . According to the diversity indices, the viral community with the most complex taxonomy was in September at the central station of the Listvyanka Tankhoy section.

The functional analysis revealed that the "Phages, prophages, transposable elements, and plasmids" category included $27.4 \%$ of classified reads in the BVP1 sample. In other viromes, the category of the first level "Cluster subsystems" comprised the main proportion. Notably, in viromes, the percentage of this category varied in a small range (12.6\%-13.6\%). Within the category (i.e. the second level), the «NULL» subcategory was the most numerous. The «NULL» subcategory can contain some incorrect assignments, which indicates either the uniqueness or the lack of the sequences with functions known in the SEED database.

In the scaffolds collected from each virome, we detected from 41 to 270 auxiliary metabolic genes (AMGs). Among them, there were, for example, DNMT1, vanY, cobS, ahbD, psbA, pbsA1, psbD, purC, cysC, nadE, etc.

In total, we collected 79 circular whole genome sequences belonging to viruses. The length of the sequences reached 80 thousand nucleotides. Among the closest relatives, there were such representatives as Ralstonia phage P-PSG-11, Methylophilaceae phage P19250A and Synechococcus phage S-CBS4.

Cluster analysis indicated that viromes from Lake Baikal formed a cluster with viromes from the world's largest (Michigan, Ontario and Erie) and the oldest (Biwa) freshwater lakes, which allowed us to identify the World's Largest Lakes (WLL) clade.

Principal components method based on the characteristics of virome sampling sites revealed that the number of bacteria and temperature have the greatest influence on the number of viral particles, which is also reasonable for the diversity of viral communities.

\section{Discussion}

Analysis of viromes from Lake Baikal has ensured for the first time the characterization of DNA viral communities in three basins of the lake. Sequences belonging to cyanophages detected in all samples comprise a large proportion in the viral population of the photic zone, indicating a great contribution of this group to the functioning of cyanobacterial communities. It is worth noting that phages belonging to the order Caudovirales, which are well represented in modern databases, predominated in the samples analysed in this study. The virome from Lake Baikal sampled in the coastal zone near the Bolshiye Koty settlement (Butina et al., 2019) formed a separate subcluster with the viromes from epilimnion of Lake Biwa, which may indicate a different composition of viral communities depending on the trophic state of the zones.

AMGs are expressed during the infection to increase and redirect the energy and resources of the host to the production of viruses; however, they are still an insufficiently studied component of biogeochemical cycles (Warwick-Dugdale et al., 2019). In this study, we detected the AMG pool in the fraction of less than 0.2 $\mu \mathrm{m}$, among which there were genes that code D1 and D2 proteins of the photosystem II reaction centre.

\section{Conclusions}

For the first time, we characterized the content of viruses in the epipelagic zone of the world's oldest and largest Lake Baikal and its three basins. The data revealed the presence of considerable amount of bacteriophages compared to the eukaryotic and archaeal viruses. The tailed bacteriophages of the order Caudovirales dominated the viromes, with prevalence of the family Myoviridae; Siphoviridae and Podoviridae were respectively the second and third dominant families. A comparative analysis of the viromes indicated their division into marine, freshwater and soil clades and allowed us to identify the WLL cluster that includes viral communities of Lake Baikal and the Great Lakes of North America.

\section{Acknowledgements}

This work was supported by State Task No. 0345-2018-0003 (AAAA-A16-116122110061-6) and the Russian Foundation for Basic Research grant No. 18-34-00513 (AAAA-A18-118032190060-5).

\section{References}

Butina T.V., Bukin Yu.S., Krasnopeev A.Yu. et al. 2019. Estimate of the diversity of viral and bacterial assemblage in the coastal water of Lake Baikal. FEMS Microbiology Letters 366. DOI: 10.1093/femsle/fnz094

Gong Z., Liang Y., Wang M. et al. 2018. Viral diversity and its relationship with environmental factors at the surface and deep sea of Prydz Bay, Antarctica. Frontiers in Microbiology 9. DOI: $10.3389 /$ fmicb.2018.02981

Potapov S.A., Tikhonova I.V., Krasnopeev A.Yu. et al. 2019. Metagenomic analysis of virioplankton from the pelagic zone of Lake Baikal. Viruses 11. DOI: 10.3390/v11110991

Skvortsov T., Leeuwe C., Quinn J.P. et al. 2016. Metagenomic characterisation of the viral community of lough neagh, the largest freshwater lake in Ireland. PLoS One 11. DOI: 10.1371/journal.pone.0150361

Warwick-Dugdale J., Buchholz H.H., Allen M.J. et al. 2019. Host-hijacking and planktonic piracy: how phages command the microbial high seas. Virology Journal 16. DOI: 10.1186/s12985-019-1120-1

Watkins S.C., Kuehnle N., Ruggeri C.A. et al. 2015. Assessment of a metaviromic dataset generated from nearshore Lake Michigan. Marine and Freshwater Research 67. DOI: $10.1071 / \mathrm{MF} 15172$

Weitz J.S., Wilhelm S.W. 2012. Ocean viruses and their effects on microbial communities and biogeochemical cycles. F1000 Biology Reports 8. DOI: 10.3410/B4-17 